\title{
A non-local anisotropic damage model for brittle materials
}

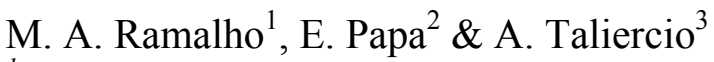 \\ ${ }^{I}$ S. Carlos School of Engineering, USP, São Carlos (SP), Brazil \\ ${ }^{2}$ Department of Health, Environment and Safety, \\ Università dell'Insubria, Varese, Italy \\ ${ }^{3}$ Department of Structural Engineering, Politecnico di Milano, \\ Milan, Italy
}

\begin{abstract}
A non-local damage model is proposed for brittle materials, such as masonry and concrete, starting from a previous proposal of the authors. The model is characterized by symmetric, second order damage tensors, which account for strain-induced anisotropy. Different laws are employed to describe the evolution of damage induced by tensile or compressive strains. The principal directions of damage remain fixed throughout any load history, and a non-rotating smeared crack model is obtained. The model overcomes some deficiencies of the previous local version, as damage at any point is computed according to the strain averaged over a suitable neighbourhood of that point, thus alleviating meshdependency effects. The model is successfully applied to the analysis of threeleaf walls tested to failure under different load conditions.

Keywords: masonry, concrete, non-local damage, anisotropy, mesh sensitivity.
\end{abstract}

\section{Introduction}

The mechanical behaviour of most engineering rocklike materials, such as concrete and masonry, is basically brittle and is characterized by strain-softening in the post-peak regime; this is especially true as far as the tensile behaviour is concerned. Strain-softening is the macroscopic manifestation of the microfracturing process that any material element experiences under increasing deformation. Microcrack coalescence leads to formation of macrocracks, and eventually failure of the material element occurs. Fracture mechanics and 
damage mechanics are both theories which try to describe the mechanical behaviour of solids beyond the onset of the cracking process. Unlike fracture mechanics, which tracks individual cracks, damage mechanics focuses on 'smeared' crack models, thus avoiding computationally heavy re-meshing procedures. This is the approach followed in this paper.

As microcracks appear in a rocklike material element, an induced anisotropy arises even in solids which are macroscopically isotropic at the original (undamaged) state. According to Kachanov [1], the effective elastic properties of the damaged material can be regarded as orthotropic with good accuracy. The constitutive model proposed by the authors and recalled in Sec. 2.1 relies on the assumption of damage-induced orthotropy.

A drawback of damage models is that, if any region exists in the body where the strain field localizes, damage localizes as well (see, e.g., [2]). In this case, the finite element results are strongly mesh dependent, and an unrealistic mechanical behaviour is described. Mesh-sensitivity effects are usually reduced assuming that damage is spread within a region (or 'process zone') whose size is supposed to be a material property [3]. The ensuing models are generally known as 'nonlocal damage models'.

The paper layout is as follows. First, in Sec. 2.1 the local damage model originally formulated by the authors is recalled. Then, the procedure proposed to make the model non-local is outlined (Sec. 2.2). Some numerical applications of the non-local damage model, implemented in two finite element codes, are shown in Sec. 3 with reference to available experimental tests on masonry walls. Finally, some critical remarks on the model are made in Sec. 4 and further developments of the research are outlined.

\section{Anisotropic damage model}

\subsection{Original (local) model}

To analyze masonry or concrete buildings and structural elements, a damage model was proposed which is briefly recalled here; readers are referred to the original paper [4] for further details. At the original (undamaged) state, any material element is supposed to be isotropic, linearly elastic: the Young's modulus and the Poisson's ratio of the material are denoted by $E$ and $v$, respectively. The damage phenomena are macroscopically taken into account through a symmetric, second-order tensor $\boldsymbol{D}$; accordingly, the damaged material is, in the most general case, orthotropic. In finite form, the nonlinear stress-strain law of the material reads:

$$
\varepsilon=C(D): \sigma
$$

where $\boldsymbol{C}=$ the fourth-order flexibility tensor of the damaged material. The eigenvalues and the normalized eigenvectors of the damage tensor will be denoted by $D_{\alpha}$ and $\boldsymbol{n}_{\alpha}(\alpha=I, I I, I I I)$, respectively. Any one of the planes of damage-induced orthotropy is somehow associated to a plane microcrack that 
forms in the solid. Once any damage direction is activated, its orientation is supposed to remain fixed throughout the rest of the stress history.

The damage process driving variable is supposed to be an equivalent strain measure, $\boldsymbol{y}=1 / 2 \boldsymbol{\varepsilon}^{2}$. As the maximum eigenvalue of $\boldsymbol{y}$ attains a critical value $\left(y_{0 T}\right.$ or $y_{0 C}$, according to the sign of the associated strain), the first damage direction $\left(\boldsymbol{n}_{I}\right)$ is activated. An additional damage direction, $\boldsymbol{n}_{I I}$, can activate in the plane orthogonal to $\boldsymbol{n}_{I}$ if the maximum direct component of $\boldsymbol{y}$, that is, $y_{h h}=\boldsymbol{n}_{h} \cdot\left(\boldsymbol{y} \cdot \boldsymbol{n}_{h}\right)$, with $\boldsymbol{n}_{h} \perp \boldsymbol{n}_{l}$, attains the damage threshold. The third possible damage direction is necessarily $\boldsymbol{n}_{I I I}=\boldsymbol{n}_{I} \wedge \boldsymbol{n}_{I I}$.

Neglecting creep-induced damage, each principal value of the damage tensor is supposed to evolve according to a law similar to that presented in [4] for concrete:

$$
D_{\alpha}=C_{H}\left(1-\frac{1}{1+A_{H}\left\langle y_{h h}-y_{0 H}\right\rangle^{B_{H}}}\right), \alpha=I, I I, I I I .
$$

Here, $\langle *\rangle$ are McAuley brackets and $A_{H}, B_{H}$ and $C_{H}$ are material parameters, which take different values according to the sign of the strain component that activates damage ( $H=T$ for tension; $H=C$ for compression).

Note that permanent (plastic) strains are disregarded in the proposed version of the model. Also, crack-closure effects (that is, the recovery of stiffness upon closure of an existing crack due to a stress reversal) are neglected. These are not deemed to be serious limitations as far as the model is applied to the simulation of tests involving monotonically increasing loads or displacements.

The proposed numerical model was implemented in two finite element codes (ABAQUS $®$ and FEAP), which are both endowed with an interface to allow users to incorporate their own constitutive law. The codes were applied to the simulation of experimental compression tests on four types of masonry prisms. The prisms either consist of mortar embedding aggregates of irregular shape or are made of stone blocks alternated with thin mortar joints. Two types of stone were employed: a sandstone tuff ('Noto' stone) and a medium-grained sandstone of very low porosity ('Serena' stone). Details on the average mechanical properties of the prisms and the experimental results can be found in [5]. In the numerical analyses, each prism was discretized by isoparametric 8-node "brick" elements. The base of the prisms is either fully restrained or simply supported.

The numerical results are shown in Figs. 1 and 2, which refer to prisms in Noto stone and Serena stone, respectively, and compared with the experimental ones. The numerical response matches the test data quite well if strains are homogenous (simply supported prism). Conversely, an excessively abrupt drop in bearing capacity is predicted if the base is fully restrained, as a consequence of the non-homogeneity of the strain field. This behaviour is typical of 'local' damage models, as the one employed so far: if any region exists in the body where the strain field localizes, damage localizes as well. The finite element results are strongly mesh dependent, and an unrealistic mechanical behaviour is described. A proposal to avoid this phenomenon is made in the following section. 


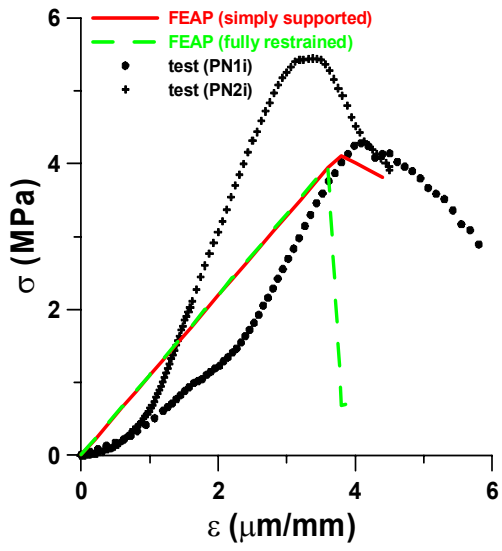

(a)

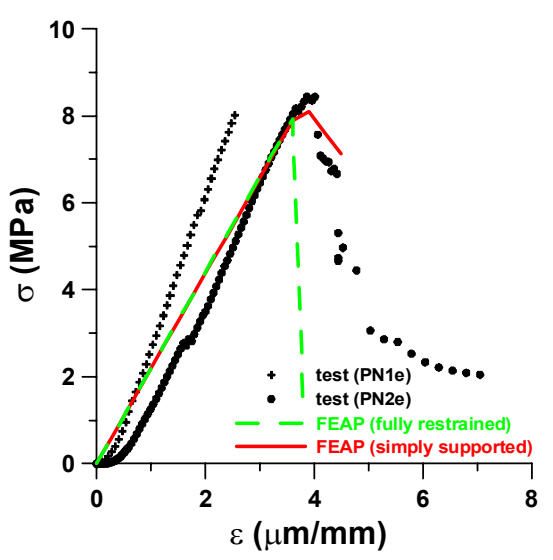

(b)

Figure 1: Experimental and numerical results for compression tests on prisms in Noto stone: (a) mortar and aggregate; (b) blocks alternated with mortar joints.

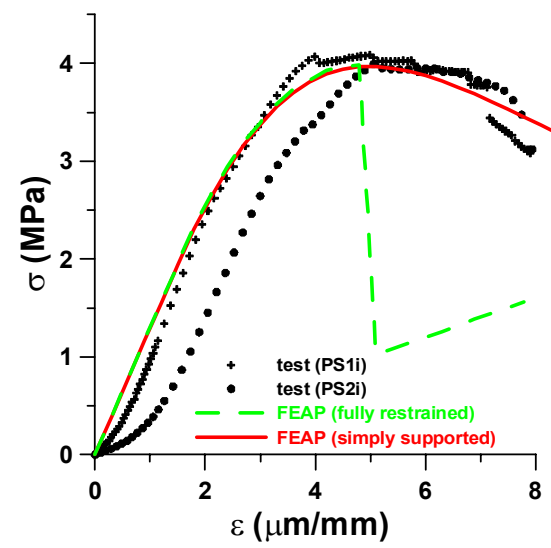

(a)

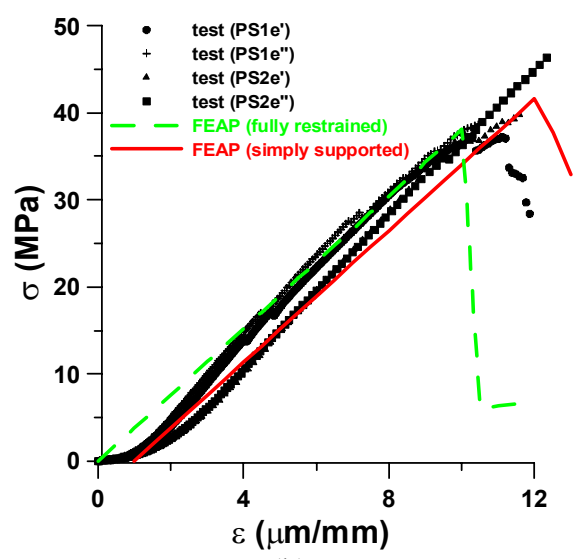

(b)

Figure 2: $\quad$ Experimental and numerical results for compression tests on prisms in Serena stone: (a) mortar and aggregate; (b) blocks alternated with mortar joints.

\subsection{Nonlocal model}

In stress analyses involving damage models the consistent damaged tangent or secant matrix is evaluated considering the local strain, that is, the strain at an integration point in FE analyses. However, for problems in which the strain field is not constant and the material exhibits strain softening, this leads to spurious results and strong mesh sensitivity (see e.g. $[6,7]$ ). Typically, the inelastic strains 
are concentrated in narrow bands, whereas the major part of the structure is nearly unstrained. This is illustrated in Figs. 8(a) and 9(a), where meshes with elements of the same height before loading are shown after deformation: strains are apparently localized in some elements, or rows of elements.

In this paper, the non-local procedure implemented in the finite element code FEAP is based on an integral-type procedure. The local strain is replaced by a weighted average of the strain field:

$$
\varepsilon_{a}(x)=\frac{\int_{V} f\left(x-x_{s}\right) \varepsilon\left(x_{s}\right) d V}{\int_{V} f\left(x-x_{s}\right) d V},
$$

where $\varepsilon_{a}$ is the average strain at the current point $x, f\left(x-x_{s}\right)$ is a weighting function that depends on the distance from $x$ to $x_{s}, \varepsilon\left(x_{s}\right)$ is the strain at any source point $x_{s}$, and $V$ is the volume of the defined neighbourhood.

The weighting function is often taken as $[7,8]$ :

$$
f\left(x-x_{s}\right)=\exp \left[-\left(\frac{x-x_{s}}{2 \lambda}\right)^{2}\right],
$$

where $\lambda$ is the internal length of the non-local neighbourhood, i.e., the diameter of a sphere centred at $x$. The value of $\lambda$ to be employed in non-local damage models is not a trivial choice. For concrete, there is a certain agreement upon taking three times the diameter of the largest aggregate [9]. No indication was found in the literature for brick or block masonry, so that the choice made in the numerical applications shown in Sec. $3(\lambda=100 \mathrm{~mm}$ for Noto stone and $60 \mathrm{~mm}$ for Serena stone) is quite arbitrary. This point is left to be addressed in the continuation of the research.

There are a few basic requirements that the averaging procedure has to meet. First, the local strain has to be replaced by the average strain only when the material is actually damaged or the results in the elastic range could be incorrect. Secondly, the model should give a realistic response even in simple loading situations, such as uniaxial tension or compression. Then, being the strain field constant, the stresses should be constant as well.

In finite element analyses, strains and stresses are usually evaluated at the integration (usually, Gauss) points. Accordingly, at any Gauss point $x_{i}$ eqn. (3) is approximated by:

$$
\varepsilon_{a}\left(x_{i}\right)=\frac{\sum_{x_{j} \in V} f\left(x_{i}-x_{j}\right) \omega\left(x_{j}\right) J\left(x_{j}\right) \varepsilon\left(x_{j}\right)}{\sum_{x_{j} \in V} f\left(x_{i}-x_{j}\right) \omega\left(x_{j}\right) J\left(x_{j}\right)},
$$

where $\omega\left(x_{j}\right)$ is the integration weight at the Gauss point $x_{j}$ and $J\left(x_{j}\right)$ is the Jacobian of the isoparametric transformation at $x_{j}$.

As FEAP detects that damage is activated at any Gauss point, a routine is called in order to identify the elements that have the centre within the non-local 
neighbourhood. The routine gets the local strains evaluated by the routines of FEAP, and returns the non-local strains according to eqn. (5), which is used to compute the secant matrix. There are two options for the weighting function. The first one is eqn. (4); the second one is $f=1$ (constant), which means to perform an arithmetic averaging over the neighbourhood.

It is worth to emphasize that the non-local strains are used only to evaluate the stiffness matrix. The stresses are computed according to the local strains, as they must be compatible with the real displacement field.

\section{Numerical applications and comparisons with experiments}

In this section some numerical simulations of experiments on three-leaf masonry wallettes tested in the laboratory are shown. Details on the specimens and the tests can be found in [5]. The outer leaves are made of stone blocks (Noto or Serena stone, see Sec. 2.1) alternated with thin mortar joints; the inner leaf is made of mortar embedding irregular pieces of the same stone that constitutes the outer leaves. Some wallettes had flat collar joints between the leaves, whereas other had keyed collar joints. Both compression and shear tests were performed. In compression tests, the lower base of the wallettes is restrained, and the top undergoes uniform increasing displacements. In shear tests, the base of the outer leaves is restrained, and the top of the inner leaf is moved downward.

In the continuation, compression tests on wallettes with flat collar joints will be labelled 'Type 1 tests'; compression tests on wallettes with keyed collar joints will be labelled 'Type 2 tests'; shear tests on wallettes with keyed collar joints will be labelled 'Type 3 tests'.

The finite element mesh employed in the numerical analyses consists of 2880 eight-node isoparametric 'brick' elements. Only one quarter of the wallettes was discretized, accounting for symmetry boundary conditions at the vertical midplanes. Each leaf was assumed to be homogeneous and the mortar joints were neglected. Perfect bonding is assumed at the collar joints. The external leaves were given 'average' mechanical properties derived from tests on the individual leaf (see also Figs. 1 and 2). The model parameters employed in the analyses are summarized in Table 1. The leaves are assumed to be perfectly bonded, except for one of the analyses (see ahead).

In Figs. 3, 4 and 5 experimental and numerical load-displacement curves are compared, for tests type 1, 2 and 3, respectively. The origin of the numerical plots was shifted to discard the initial stage of the laboratory tests, where an adjustment takes place between machine platens and specimens. The numerical results obtained with the local version of the damage model were already presented in [6].

In the simulation of the tests on the specimen in Noto stone both FEAP and ABAQUS predict the peak load and the corresponding displacement with fair accuracy (Fig. 3(a)). Regarding the post-peak softening behaviour, however, only FEAP, where a non-local damage procedure is implemented, yields good results; on the contrary, ABAQUS, in which the local damage model is implemented, predicts an excessively brittle response. For Serena stone the 
results are not conclusive, as the specimen did not fail during the test: the relevant load-displacement plot does not exhibit any significant non-linearity (Fig. 3(b)).

Table 1: $\quad$ Model parameters employed in the FE analyses.

\begin{tabular}{|l|l|l|l|l|l|}
\cline { 3 - 6 } \multicolumn{2}{c|}{} & \multicolumn{2}{c|}{ Noto stone } & \multicolumn{2}{c|}{ Serena stone } \\
\cline { 3 - 6 } \multicolumn{2}{c|}{} & outer leaf & inner leaf & outer leaf & inner leaf \\
\hline $\begin{array}{l}E \\
(\mathrm{MPa})\end{array}$ & FEAP & 2200 & 1100 & 3800 & 1300 \\
\cline { 2 - 6 } & Abaqus & 2500 & 1800 & 3800 & 1875 \\
\hline \multirow{3}{*}{$y_{0 C}$} & FEAP/Abaqus & 0.15 & 0.15 & 0.15 & 0.15 \\
\hline \multirow{3}{*}{$A_{C}$} & FEAP & 0.001 & 0.001 & 0.1 & 0.0001 \\
\cline { 2 - 6 } & Abaqus & $0.5 \mathrm{E}-6$ & $0.5 \mathrm{E}-6$ & $0.5 \mathrm{E}-6$ & $0.5 \mathrm{E}-6$ \\
\cline { 2 - 6 } & FEAP & $6.68 \mathrm{E}+6$ & $1.78 \mathrm{E}+6$ & $23.42 \mathrm{E}+6$ & $0.545 \mathrm{E}+6$ \\
\hline \multirow{3}{*}{$B_{C}$} & Fbaqus & $0.34 \mathrm{E}+6$ & $0.30 \mathrm{E}+6$ & $1.0 \mathrm{E}+6$ & $0.50 \mathrm{E}+6$ \\
\cline { 2 - 6 } & Abaqus & 1.27 & 1.2 & 1.5 & 1.2 \\
\hline \multirow{2}{*}{$C_{C}$} & FEAP & 1.2 & 1.11 & 1.3 & 1.2 \\
\cline { 2 - 6 } & Abaqus & 2.8 & 2.0 & 6.0 & 1.05 \\
\hline
\end{tabular}

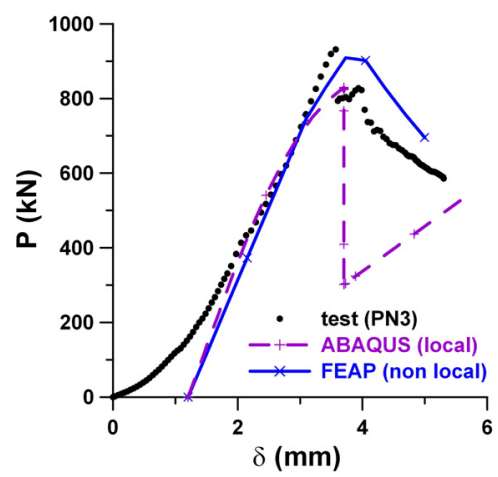

(a)

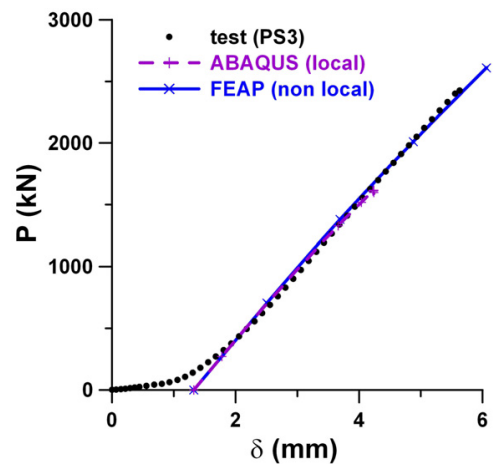

(b)

Figure 3: Load-displacement plots for Type 1-tests: (a) Noto stone, (b) Serena stone.

Similar remarks apply to the simulation of Type 2 tests (Fig. 4). For Serena stone, the local damage model implemented in ABAQUS predicts a peak load which is not matched by the experimental plot, as the specimen did not fail during the test; this is not the case with FEAP (see Fig. 4(b)).

Finally, regarding Type 3 tests (Fig. 5) FEAP overestimates the peak-load for specimens both in Noto and Serena stone. Also, both codes overestimate the stiffness of the specimens in Serena stone (Fig. 5(b)). Both results are likely to be due to the assumption of perfect bonding at the collar joints. Indeed, the bonding between stones and mortar is negligible, as indicated by the separation 
of the leaves which took place in the tests at very low load values [5]. To check the validity of this assumption, a numerical analysis was performed with ABAQUS assuming a Coulomb's-type law at the collar joints (friction coeff. = 0.866). The results are shown in Fig. 5(b): note that, whereas the stiffness of the specimens is correctly matched, the peak load is definitely underestimated, indicating that the interfacial cohesion cannot be completely neglected. Thus, it would be extremely important to employ an interface element to correctly take the imperfect bonding between the leaves into account.

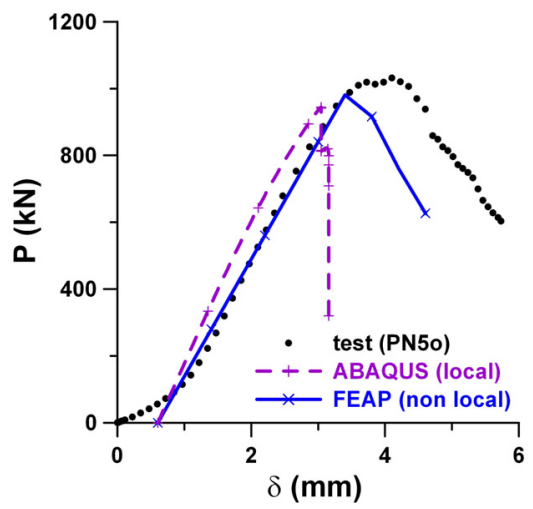

(a)

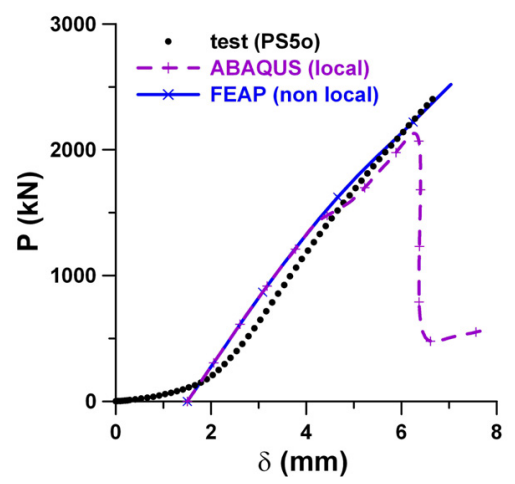

(b)

Figure 4: Load-displacement plots for Type 2-tests: (a) Noto stone, (b) Serena stone.

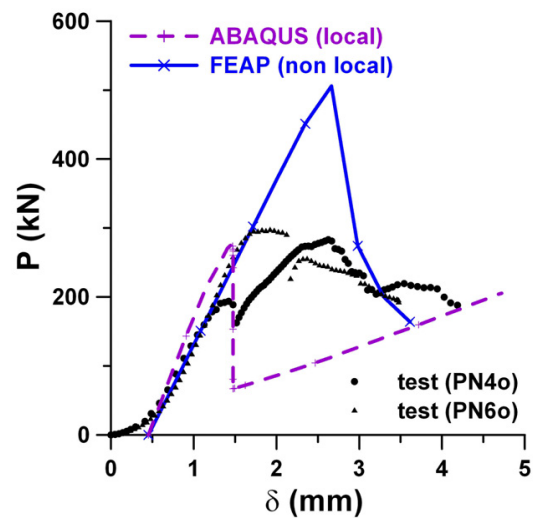

(a)

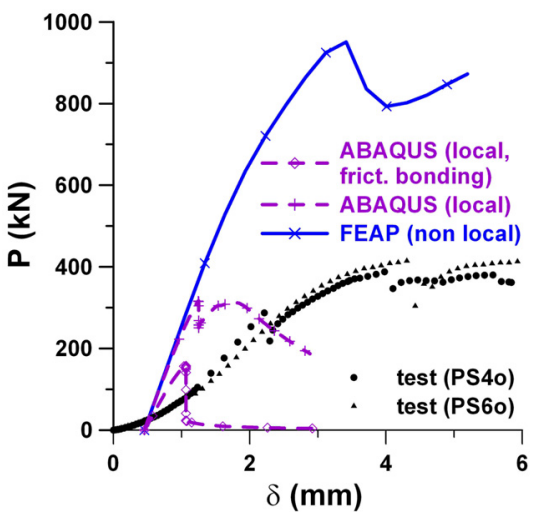

(b)

Figure 5: Load-displacement plots for Type 3-tests: (a) Noto stone, (b) Serena stone.

Figures 6 and 7 show the contour plots of the axial stress (in MPa) obtained in the post-peak regime for specimens in Noto stone subjected to type 1 test and 
for specimens in Serena stone subjected to type 3 test, respectively. The plots are drawn on the deformed FE meshes. When the local model is employed, damage (and strains) localize either in a strip of elements (Fig. 6(a)) or just below the loaded side of the model (Fig. 7(a)), which makes the numerical solution strongly mesh-dependent. The use of the non-local damage model definitely regularizes the obtained deformation mode (see Figs. 6(b) and 7(b)). Note, however, that in type 3-tests the experimental crack pattern, which mostly runs along collar joints [5], is not matched by the numerical predictions.

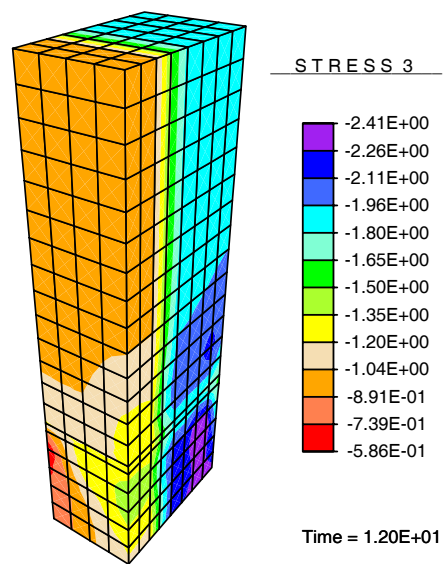

(a)

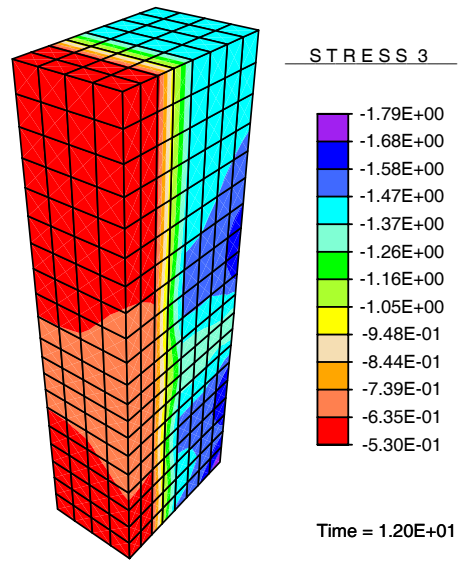

(b)

Figure 6: Type 1-tests on walls in Noto stone: contour plots for the axial stress in the post-peak regime and deformed mesh obtained with the local (a) and the nonlocal (b) damage model.

\section{Concluding remarks and future perspectives}

The use of a non-local damage model allows the bearing capacity and the postpeak response of several masonry specimens tested in the laboratory to be captured with fair accuracy. This could not be achieved with the original (local) version of the proposed damage model, as a consequence of the localization of strains and damage in a band of finite elements. The non-local procedure prevents such localization and alleviates the mesh-sensitivity of the numerical results.

Future developments of the research will be addressed to the formulation of brittle interface elements, to allow for failure modes involving the collar joints: this was the dominant failure mode observed in the shear tests on the three-leaf walls considered in Sec. 3. Finally, as the proposed model is aimed at the safety assessment of historical massive masonry buildings, creep-induced damage will also be taken into account in order to predict possible structural failures under sustained loads of high intensity. 


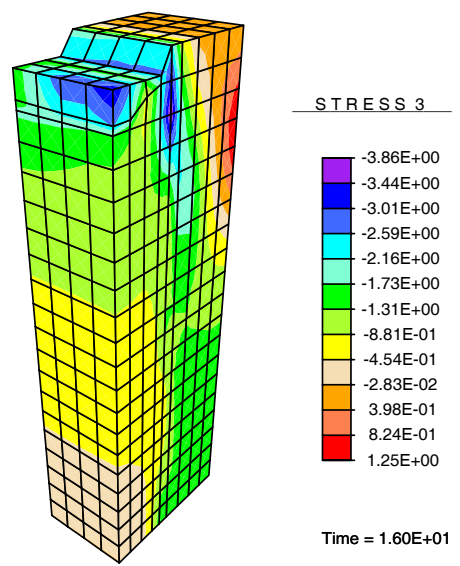

(a)

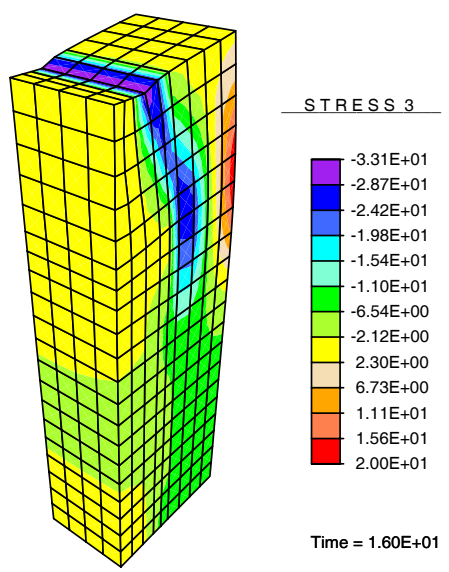

(b)

Figure 7: $\quad$ Type 3-tests on walls in Serena stone: contour plots for the axial stress in the post-peak regime and deformed mesh obtained with the local (a) and the nonlocal (b) damage model.

\section{References}

[1] Kachanov, L.M., Elastic solids with many cracks: a simple method of analysis. Int. J. Solids Structures, 23, pp. 23-43, 1987.

[2] Comi, C. \& Perego, U., Fracture energy based bi-dissipative damage model for concrete. Int. J. Solids Structures, 38(36-37), pp. 6427-6454, 2001.

[3] Lemaitre, J., A Course on Damage Mechanics. Springer-Verlag, Berlin, 1992.

[4] Papa, E. \& Taliercio, A., A damage model for brittle materials under nonproportional monotonic and sustained stresses. Int. J. Num. Anal. Methods Geomech., 29(3), pp. 287-310, 2005.

[5] Anzani, A., Binda, L., Ramalho, M.A. \& Taliercio A., Historic multi-leaf walls: experimental and numerical research. Masonry International, 18(3), pp. 101-114, 2005.

[6] Bažant, Z.P., Instability, ductility, and size effect in strain-softening concrete. J. Engng. Mech. Div. (ASCE), 102(2), pp. 331-344, 1976.

[7] Jirásek, M., Nonlocal models for damage and fracture: comparison of approaches. Int. J. Solids Structures, 35(31-32), pp. 4133-4145, 1998.

[8] Rodriguéz-Ferran, A., Morata, I. \& Huerta, A., Efficient and reliable nonlocal damage models. Comput. Methods Appl. Mech. Engrg., 193, pp. 3431-3455, 2004.

[9] Mazars, J. \& Pijaudier-Cabot, G., Damage localization analyzed as a crack propagation? Fracture and Damage in Quasibrittle Structures: Experiment, modelling and computer analysis, eds. Z.P. Bažant et al., E\&FN Spon, London, pp. 145-157, 1994. 